Aage A. Hansen-Löve

\title{
Frühsowjetische Avantgardeprosa: Ornament statt Verbrechen
}

\section{Die Situation - „Serapionsbrüder“ zwischen Ost und West}

Inmitten der heftigsten Bürgerkriegswirren des Jahres 1919 hatte sich gemeinsam mit einer bunten Schar junger Autoren der Hispanistik-Student Lev Lunc in dem von Maxim Gorkij unterstützten „Haus der Künste“ durch die Bürgerkriegsjahre Petrograds gerettet. Man fühlte sich dort wie in einer Arche Noah, in der sich ein breites Spektrum von Erzähl-Figuren - vom braven Haustier bis zum Paradiesvogel - die kargen Zimmer und Essensrationen teilte.

Aus dieser losen Gruppe, die sich dann um 1921 nach E. T. A. Hoffmann „Serapionsbrüder“ von Petrograd nannte, ${ }^{1}$ sollte die Blüte der Prosaavantgarde der jungen Sowjetunion hervorgehen: Sie umfasste unter anderen Viktor Šklovskij, Evgenij Zamjatin, Konstantin Fedin, Lev Lunc, Michail Slonimskij, Vsevolod Ivanov, Michail Zoščenko, Veniamin Kaverin.

Eine sehr lebendige Schilderung des stürmischen Lebens im „Haus der Künste“ liefert Viktor Šklovskijs Die sentimentale Reise, erstmals publiziert 1923 in Berlin, ${ }^{2}$ von der noch die Rede sein wird. Die erste Sitzung der Gruppe fand Anfang Februar 1921 statt - ohne Wahlen oder Anführer, ohne Programm und gerichtet gegen eine jede Ideologie und Weltanschauungsprosa. Zentral waren die freundschaftliche Verbindung der blutjungen Autoren und ihre geradezu naive und provokante Unabhängigkeit von den literarischen Machthabern.

Von Anfang an zeigte sich auch bei den Serapionsbrüdern die traditionelle Gespaltenheit der russischen Literatur, ja Kultur insgesamt, in ,Ostler‘ und ,Westler‘: Die einen orientierten sich an der übermächtigen Tradition der handlungsschwachen ornamentalen Prosa des russischen Realismus und vor allem der symbolistischen

\footnotetext{
1 Siehe dazu auch Viktor Schklowski: Die Serapionsbrüder [1921], in: Fritz Mierau (Hrsg.): Die Erweckung des Wortes. Essays der russischen Formalen Schule, Leipzig 1987, 59-61. Der gleichnamige Sammelband von 1921 erschien in der Übersetzung von Gisela Drohla (Hrsg.), Die Serapionsbrüder von Petrograd, Frankfurt a. M. 1963.

2 Viktor Šklovskij: Sentimental'noe putešestvie. Vospominanija. 1917-1922, Berlin 1923 (dann modifiziert: Leningrad 1924); deutsch: Viktor Schklowskis Sentimentale Reise, Frankfurt a. M. 1964, 262-272 und a. a. O.; zu den theoretischen Hintergründen vgl. Aage Hansen-Löve: Der russische Formalismus. Methodologische Rekonstruktion seiner Entwicklung aus dem Prinzip der Verfremdung, Wien 1978, 515-537.
} 
Erzählgenres, die anderen verstanden sich als ,Westler ' mit Vorliebe für eine Handlungsprosa mit starkem Sujet, mit Intrigen und Spannung.

Lev Lunc war es denn auch, der in jugendlichem Ungestüm die für die Epoche der heraufziehenden Staatskunst und ihrer ideologischen Zwänge provokante Losung ausgab, die Prosaavantgarde müsse sich „Nach Westen!“ orientieren, ${ }^{3}$ zumal an der anglo-amerikanischen Spannungs- und Trivialliteratur. Diese passte durchaus in die Frühphase der NEP-Periode der 20er Jahre ${ }^{4}$ mit ihrer Begeisterung für die TechnikUtopien Amerikas, für den Taylorismus und die modern times.

Auch Evgenij Zamjatins poetische Welt ist aufgespannt zwischen zwei diametral entgegengesetzten Polen, die sich mit der Ost-West-Spannung der von ihm (mit-)begründeten Serapionsbrüder von Petrograd deckt: Archaismus bzw. Neoprimitivismus, ${ }^{5}$ verkörpert durch die ,Ostler‘ (Vsevolod Ivanov, Nikolaj Nikitin u.a.) und Utopismus bzw. Urbanismus, vertreten von den ,Westlern' (Lev Lunc, Veniamin Kaverin, Viktor Šklovskij u. a.). Wie im Falle der russischen Avantgarde der 10er Jahre konnte ein und derselbe Autor beiden Tendenzen folgen - so auch Zamjatin mit seiner neoprimitivistischen Bürgerkriegsprosa um 1920 und seinen radikal primitivistischen Erzählungen Die Höhle oder Hochwasser, die seine ,westlerische“ Antiutopie Wir mit dem atavistischen Unterbewussten des Ostens konfrontieren. ${ }^{6}$

Ähnliches lässt sich übrigens von Vsevolod Ivanov sagen, von Veniamin Kaverin oder Viktor Šklovskij selbst, der auch beständig zwischen sujetloser und sujethafter Prosa pendelte bzw. beide narrativen Medien miteinander verschränkte - so etwa in seinem literarischen Hauptwerk Die sentimentale Reise, ${ }^{7}$ das nicht zufällig und auf höchst ironische Weise an den Vater der antigenerischen Prosa anschließt.

3 Lev Lunc: Warum wir Serapionsbrüder sind, in: Gisela Drohla (Hrsg.): Die Serapionsbrüder von Petrograd, Frankfurt a. M. 1963, 7-12. Zur Sujet-Prosa der 20er Jahre vgl. Aage Hansen-Löve, Formalismus (Anm. 2), 510 ff.; zur „ornamentalen Prosa“ jener Periode ebd., 530 ff. und Wolf Schmid: Ornamentales Erzählen in der russischen Moderne. Čechov - Babel’ - Zamjatin, Frankfurt a. M. u. a. 1992. Vgl. die deutsche Ausgabe der Schriften von Lev Lunc: Die Affen kommen, Erzählungen, Dramen, Essays, Briefe, Münster 1989 (hier: Warum wir Serapionsbrüder sind, 257-261; Nach Westen!, 267-280).

4 NEP = „Neue Ökonomische Politik“ (1921-1927). Ausführlich dazu: Manfred Hildermeier: Geschichte der Sowjetunion 1917-1991, München 1998, 157-363.

5 Aage Hansen-Löve: Über das Vorgestern ins Übermorgen. Neoprimitivismus in Wort- und Bildkunst der russischen Moderne, Paderborn 2016, 417-425.

6 Deutsche Übersetzung in der Ausgabe Jewgeni Samjatin: Erzählungen 1917-1928, Leipzig/Weimar 1991 (russisch: Evgenij Zamjatin: Izbrannye proizvedenija, Moskau 1989, mit einem Vorwort von Viktor Šklovskij). Zamjatins Roman My (1920; deutsch: Jewgeni Samjatin: Wir. Roman, Leipzig 1991) ist in diesem Zusammenhang weniger als (Anti-)Utopie relevant, sondern vielmehr als eine vielschichtige $\mathrm{Pa}$ rodie parawissenschaftlicher und poetologischer Konzepte der (primitivistischen) wie futuristischen Avantgarden. Zamjatins Roman realisiert idealtypisch die radikale Perversion des Reprojektions-Prinzips, das in der inversiven Formel ,Vom Übermorgen ins Vorgestern' resultiert. Vgl. auch Hansen-Löve: Schwangere Musen - Rebellische Helden. Antigenerisches Schreiben, Paderborn 2018, 604-614. 7 Šklovskij, Sentimentale Reise (Anm. 2); dazu: Hansen-Löve, Formalismus (Anm. 2), 530-537; Schmid: Ornamentales Erzählen (Anm. 3). 
Beide Pole der Prosaavantgarde der 20er Jahre existierten nicht in Reinkultur, sondern immer wieder in Überschneidungen, mehr noch: Diese verfolgen das eigentliche Ziel, die Hinter- und Untergründe der urbanen Welt (in Extremsituationen wie Bürgerkrieg und Blockade) zu zeigen, die unter dem dünnen Firnis der Zivilisation und Modernität lauert. Umgekehrt drohten aber auch die utopischen und avantgardistischen Aspekte der Revolutionsära in ihrer technischen und mechanischen Brutalität.

Eine vergleichbare Mischung aus mythischen und aktuellen Motiven findet sich seit 1900 bei den Symbolisten - zumal bei Brjusov, Blok und Belyj, deren stilisiertes Skythentum und Asienfaszination immer wieder mit Motiven des zeitgenössischen Urbanismus gekreuzt werden. Eine ähnliche Polarisierung in eine neoprimitivistische und eine futuristisch-konstruktivistische Richtung (und der Montage beider in ein und demselben Werk) entwickelte die postsymbolistische Avantgarde (wobei ,post ${ }^{\star}$ weniger zeitlich als systematisch gemeint ist, da etwa in den 10er und 20er Jahren beide Richtungen gleichzeitig am Werk waren).

Das entsprechende Textkorpus der ,ornamentalen Prosa‘ jener Jahre ist so umfangreich, dass Lev Lunc - einer der Haupttheoretiker der Petrograder Serapionsbrüder im Geiste Šklovskijs und der jungen Formalisten in die Klage ausbrechen konnte, die entgegengesetzte Dominante - eine sujet- und spannungsorientierte Prosa ${ }^{8}$ - wäre peinlich unterentwickelt geraten. Die russischen Autoren verständen sich nicht auf die Intrige, ja sie wären zur einfachsten Kriminalhandlung unfähig und neigten zu Ornament statt Verbrechen:

Im Westen gibt es von jeher eine gewisse Literaturgattung, die in Rußland als unseriös, um nicht zu sagen schädlich, angesehen wird. Das ist die sogenannte Abenteuerliteratur. [...] Und wir Russen, wir verstehen nicht, mit der Handlung umzugehen, wir ignorieren sie und deshalb verachten wir sie. [...] Psychologisieren [wie die Russen] kann auch ein Neger, doch eine Handlung aufbauen kann nur ein Schriftsteller, der eine große Schule durchlaufen hat. [...]

Deshalb - nach Westen!

Deshalb - auf die Schulbank, lernt das Abc!

Fangt von vorne an! ${ }^{9}$

8 Gemeint ist hier aus der Sicht Viktor Šklovskijs eine stark markierte Handlungsstruktur zumal in der für die Prosaavantgarde der frühen 20er Jahre typischen Gattungen der metafiction. Vgl. dazu Erika Greber: The Metafictional Turn in Russian Hoffmannism, in: Essays in Poetics 17.1. (1992), 1-34; dies.: Metafiktion - ein ,blinder Fleck' des Formalismus?, in: Poetica 40 (2008), 43-71.

9 Lev Lunc, Nach Westen! (Anm. 3), 276. 


\section{Formalistische Erzähltheorie: Sujetlose/sujethafte Prosa}

$\mathrm{Zu}$ den radikalsten Theoriebeständen des jungen Formalismus um 1920 gehört zweifellos die frühe Erzähltheorie, die sich primär an der Differenz von ,Fabel' (fabula) und ,Sujet“ (sjužet) orientierte. In der fabula dominiert der kausal-empirisch rekonstruierbare und dem jeweiligen Wahrscheinlichkeitsmodell (Jakobson) folgende ordo naturalis (für Jakobson das jeweils herrschende „Wahrscheinlichkeitsmodell“), ${ }^{10}$ womit eigentlich ein ordo culturalis gemeint ist. ${ }^{11}$ Eine solche Fabel gibt es freilich nur in einem annähernd realistischen Erzählen, das sich auf pragmatische Kontexte und konventionelle Handlungsabläufe verlässt, ohne die der Erzähltext unverständlich wäre. Diese als vorgegeben gedachte Ordnung (die zeitliche, logische, alltägliche Reihung von Motiven und Motivationen) wird im jeweiligen narrativen Sujet deformiert, verfremdet und auf spezifische Weise umgebaut. Es wird hier die ursprünglich konstitutive Primärfunktion der Verfremdung in sekundäre, abgeleitete Differenzierungen verlagert, die aus den Regeln der Text-Syntagmatik, einer Art narrativen Syntax, resultieren. Diese ist regulativ und normbildend im Rahmen eines generellen narrativen Codes (der Erzähl-Sprache und ihrer sukzessiven Motiv-Präsentation), der wiederum in eine spezielle textuelle Performanz (im konkreten Einzeltext) und eine spezielle Realisierung im Rezipienten (durch die Orientierung auf außertextuelle bzw. kulturelle Kontexte) perspektiviert ist. Das Sujet ist für die Formalisten kein Thema, nichts Stoffliches - sondern bezeichnet ausschließlich die Regeln der Text-Syntagmatik, eine Art narrative Syntax.

Hier haben wir es dann - analog zur Vers-Rede - mit der Erzähl-Rede zu tun. In der frühen, vor allem von Šklovskij entwickelten Sujettheorie (zusammengefasst in seiner Theorie der Prosa, 1925 verfasst, erweitert 1929) ging es vor allem um die Verfremdungs-Effekte einer maximalen Differenz zwischen vorgegebener bzw. mitgedachter fabula-Ordnung und der konkreten, an generellen Regeln orientierten narrativen Syntax (Sujet).

Erzählen in der Moderne, besonders aber in Zeiten der Avantgarde, war immer eine kritische, ja polemische Auseinandersetzung mit den fiktionserzeugenden Potenzialen des Narrativen - oder anders gesagt: Eine jede Avantgarde-Prosa strebte nach der Verfremdung, Demontage und Neumontage des Erzählens, sei es der Ereignisse

10 Vgl. Roman Jakobson: Über den künstlerischen Realismus [1921], in: Jurij Striedter (Hrsg.): Texte der russischen Formalisten, Bd. 1, München 1969, 372-391.

11 Zur Unterscheidung von Fabel und Sujet im Rahmen der formalistischen Erzähltheorie vgl. Hansen-Löve, Formalismus (Anm. 2), 238-260; Wolf Schmid, Elemente der Narratologie, Berlin 2014, 205 ff.; Wolf Schmid: ,Fabel‘ und ,Sujet', in: ders. (Hrsg.): Slavische Erzähltheorie. Russische und tschechische Ansätze, Berlin/New York 2009, 1-45. 
einer Handlung, der eingesetzten Diskurse oder einer identifizierbaren Erzählperspektive. Immer ging es um einen Effekt der Defiktionalisierung mit dem Ziel einer Des-Illusionierung und Ent-Täuschung jener narrativen Schablonen einer Alten Welt, die sich der Prosa des Lebens ebenso beugte wie den Konventionen einer Romanwelt, die mit der Herrschaft des ancien régime auf den Abfallhaufen der Literaturgeschichte gelandet war.

\section{Parteinahme der Formalisten für die Sujet-Prosa}

Šklovskij und die anderen jugendlichen Vertreter des russischen Formalismus an der Wende zu den 20er Jahren wollten sich nicht prinzipiell für einen der beiden Prosatypen entscheiden. Der „Auftrag“ (zakaz) des zeitgenössischen Genre-Systems war primär die entblößte, ja provokante Demonstration eines jeweiligen Extrem-Typs, also totale Sujethaftigkeit und totale Sujetlosigkeit, als Avantgarde-Konzepte - oder aber eine Kombination aus beiden in einem akzentuierten dritten Typus, der die prägnante Sujethaftigkeit mit einer Material-Montage im Sinne der „Faktenliteratur“ kombinierte. ${ }^{12}$ Eben dies gilt für serielle Großtexte wie Šklovskijs Sentimentale Reise.

Auch in seiner Theorie der Abenteuer-Erzählung bzw. des Abenteuerromans im Rahmen seiner Theorie der Prosa ging es Šklovskij primär um die verfremdende, schockierende Rehabilitierung eines den russischen Erzähl-Kanon (Realismus, epische Breite, Handlungsschwäche) negierenden und provozierenden Kriminal- und Abenteuergenres - sei es nach dem Modell von Conan Doyle oder des alten Abenteuergenres im Geiste Cervantes'. ${ }^{13}$ Beiden hat Viktor Šklovskij bahnbrechende Studien in seiner Theorie der Prosa gewidmet.

Im Mittelpunkt stand das Interesse an Sujet-Verfremdung und nicht an einer ontologischen Definition einer generischen Erzähl-Gattung, die favorisiert oder kritisiert werden sollte. In diesem eminent avantgardistischen Verständnis von Genre ist die Intention auf das Sujet immer schon etwas Parodistisches, da die im Realismus psychologisch, sozial oder ideologisch reich bestückten Motive und Motivationen der Protagonisten auf das reine Handlungsskelett reduziert werden.

Šklovskij unterscheidet in seiner frühen Prosatheorie - auch mit Blick auf die aktuelle Situation der (avantgardistischen) Erzählgenres der frühen 20er Jahre - zwischen Sujet-Verfremdungen mit Hilfe markanter syntagmatischer Umstellungen in massiv

12 Ausführlich zur Position der Formalisten im Rahmen des Genre-Systems der 20er Jahre vgl. Hansen-Löve, Formalismus (Anm. 2), 510-570.

13 Vgl. dazu Aage Hansen-Löve: „Wir sind zur einfachsten Kriminalhandlung unfähig.“ Experimentelle Schundliteratur der russischen 20er Jahre, in: Martin von Koppenfels, Manuel Mühlbacher (Hrsg.): Erzählformen des Abenteuers, Paderborn 2019, 237-261. 
handlungsorientierten Genres (ostrosjužetnye zanry) ${ }^{14}$ wie Kurzgeschichten, Novellen, Kriminal- und Abenteuerromanen und solchen in bewusst sujetschwachen Genres, die dem Prinzip der „Sujetlosigkeit“ (bessjužetnost’) folgen.

In diesem Sinne ist für Šklovskij die „Sujetlosigkeit“ in der Prosa mit der „Gegenstandslosigkeit“ in der Malerei (eines Malevič) homolog. ${ }^{15}$ Ergänzend könnte man sagen, dass wiederum die zugespitzte Sujethaftigkeit mit der abstrakten Malerei und den Artefakten des Konstruktivismus vergleichbar sei. Auch hier werden die konstruktiven Prinzipien des Textes, der Struktur des Artefakts analog. Die konstruktiven Formen werden zum Hauptinhalt des Romans: „Die Sujet-Form ist so eingesetzt wie die realen Gegenstände in den Bildern Picassos eingesetzt sind [...] Die Einstellung auf die Tatsache dieses Verfahrens selbst - d. h. die Wahrnehmung seiner Form - ist der Inhalt des Kunstwerks."16 Die Option der Formalisten für die demonstrativ sujethaften Genres war also nur die „eine Hälfte der Wahrheit“ - oder genauer: einer der hoch reflektierten narrativen „Pole“. Die andere Hälfte war ihr genaues Gegenteil: die Favorisierung der sujetlosen Prosa.

\section{Angewandte Literaturwissenschaft: Formalistische Prosa}

Die Aufträge an Kunst und Literatur kommen aus einer solchen Sicht nicht direkt aus der Gesellschaft oder den ideologischen Apparaten, sondern aus den objektiven Erfordernissen des Gattungssystems, aus der Aktualität des herrschenden Kulturdiskurses: Die ,literarische Gegenwart', das dynamische ,Zeitgenossentum' (sovremennost') und seine Interessenslage sollte entkoppelt werden vom Parteigenossentum und den Kommandostellen der offiziellen Kulturpolitik. Es sind objektiv nachvollziehbare, diachron herleitbare und synchron wirksame Gesetzmäßigkeiten, die ein soziales oder kulturelles Faktum zu einem , literarischen Faktum' machen. Heute würden wir sagen: dieses wird projektiv ,konstruiert‘, konzeptualisiert, ,erfunden‘; damals sprach man von einer Konstruktion im Sinne von Machen, jetzt aber nicht mehr ein Montage-Bau

14 Wörtlich bedeutet dies ,scharfsujethafte' Genres, d.h. solche, in denen das schwach motivierte Handlungsgerüst als solches entblößt präsentiert wird.

15 Viktor Šklovskij: Chod konja, Berlin 1923, 99-100; dazu: Hansen-Löve, Formalismus (Anm. 2), 254 f. Zur Differenzierung von Ungegenständlichkeit (Malevičs) und Abstraktion (Kandinskijs) vgl. Aage Hansen-Löve: Die Kunst ist nicht gestürzt. Das suprematistische Jahrzehnt, in: Kazimir Malevič: Gott ist nicht gestürzt! Schriften zu Kunst, Kirche, Fabrik, hrsg., eingeleitet und kommentiert von Aage Hansen-Löve, München 2004, 255-603, hier: 353-359; Tanja Zimmermann: Abstraktion und Realismus im Literatur- und Kunstdiskurs der russischen Avantgarde, Wien/München 2007, 54-60.

16 V. Šklovskij: Evgenij Onegin. Puškin i Stern, in: Očerki po poètike Puškina, Berlin 1923, 199-220, hier: 211. 
auf der ,grünen Wiese‘, sondern eine Art Selbstorganisation unter der Wirkung jener Kraftfelder, die in der aktuellen Kulturszene quasi naturgesetzlich auftreten.

Das radikal hypothetische Entwicklungsgesetz der formalistischen Evolutionstheorie tritt in ein Rückkoppelungsverhältnis mit den synchronen Interessenslagen, die ihrerseits bestimmen, was ,interessant' und aktuell ist und was nicht. Diese Etablierung von wechselnden Relevanzen ist einerseits durchwegs relativ und gegen eine jede Ontologie und einen jeden Essenzialismus gerichtet; zugleich aber sind die nunmehr ins Auge fallenden Gewohnheitsregeln der aktuellen Situation nicht weniger stringent als die kulturelle Grammatik oder überhaupt jene Codes, um die es den frühen Formalisten primär zu tun war. Sei es, um sie zu knacken, sei es, um neue zu etablieren. Es ging darum, den soziologischen bzw. ideologischen Determinismus einer kruden Widerspiegelungstheorie und ihrer pseudorealistischen Mimesis durch ein falsifizierbares Regelwerk zu ersetzen, das nicht nur historisch rekonstruierbar sein sollte, sondern auch - wie in den „harten Wissenschaften“ - prädiktabel zu sein hatte: Eine Gesetzmäßigkeit der Biosphäre war ja auch nur dann eine solche, wenn sie nicht nur für die Vergangenheit Geltung hätte, sondern auch gegenwärtige und vor allem zukünftige Situationen regulierte.

Was die harten Naturwissenschaften für ihre Biosphäre postulierten, sollte auch für die Semiosphäre gelten, mehr noch: die Literaturwissenschaftler, der Formalismus zumal, griffen eigenhändig in den literarisch-künstlerischen Prozess ihrer Gegenwart ein und erweiterten die Position der Bewertung synchroner Werke durch eigenes literarisches Schaffen, indem sie nicht etwa bloß Erzähltheorie betrieben, sondern gleich selbst Romane, Erzählungen und Filmszenarien schrieben.

\section{Sujetlosigkeit und Prosa-Montage}

Somit ist es nur konsequent, dass die Formalisten, allen voran Šklovskij, Laurence Sternes Tristram Shandy als den „typischsten Roman der Weltliteratur“ hochleben ließen. ${ }^{17}$ Damit war die sujetlose Prosa der Abschweifungen und permanenten Aufschübe gleichfalls zum authentischen Typus der Sujet-Verfremdung im Sinne der Prosa-Avantgarden erklärt: Die traditionellen raum-zeitlichen Kontinua der fabula wurden hier - nicht selten auch im wörtlichen Sinne - ,gesprengt', dissoziiert, disseminiert und zu komplexen Texturen umgetextet (wie in der Prosa der Symbolistengeneration) oder zu neuen Konstrukten umgeschraubt wie in der fast gleichzeitig entstehenden Montage-Prosa. Schließlich konnte so die Leitmotiv-Technik ${ }^{18}$ der

17 Viktor Šklovskij: Theorie der Prosa [1925] Frankfurt a. M. 1964, 162.

18 Dagmar Burkhart: Leitmotivik und Symbolik in Andrej Belyjs Roman Peterburg, in: Welt der Slaven 3 (1964), 277-323. 
narrativen Symphonien des Symbolismus um 1900 mit der kinematographischen wie kubo-futuristischen Schnitt-Technik einer Stummfilm-Prosa verknüpft werden. Dabei konnten triviale Spannungs-Szenarien der Abenteuerliteratur ebenso zum Zug kommen wie die Materialschlachten einer aufs Faktische entnarrativierten Prosa in der Faktenliteratur.

\section{Sujethafte und sujetlose skaz-Prosa: charakterisierende und ornamentale Erzähl-Rede}

Neben der Problematik der narrativen Sujets - also einer genuin syntagmatischen Struktur-Analytik - stellte sich parallel dazu (zumal für Boris Ejchenbaum und Viktor Vinogradov) die Frage nach der Erzähl-Rede, also der Stilistik und der Perspektivierung der Präsentation der Erzählung. ${ }^{19}$ Zusammengefasst sind die formalistischen Theorien zum ,Redeverhalten` der Figuren bzw. Erzähler unter dem Konzept ,Theorie des skaz', wobei der Begriff des skaz die Simulation der mündlichen Rede eines konkreten Sprechers im Rahmen des Schrift- bzw. Druckmediums bezeichnet. Es geht hier also auch um die intermediale Frage, auf welche Weise die schriftliche Literatur (pis'mennost') in der Lage ist, Verfahren und Merkmale der ,Mündlichkeit‘ (ustnost') zu markieren bzw. in einer stilisierten Form nachvollziehbar zu machen.

Gerade für die skaz-Technik gab es in der russischen Prosa von Gogol, Dostoevskij und Leskov über die Symbolisten (Andrej Belyj) bis zu den Vertretern des, Östlichen Flügels‘ der Petrograder Serapionsbrüder bzw. der zeitgenössischen ,ornamentalen Prosa' (Isaak Babel, Boris Pil'njak, Vsevolod Ivanov etc.) eine reiche Tradition, die analog zur Bindung des frühen Formalismus an die futuristische Avantgarde-Dichtung - eine vitale Prosa-Avantgarde als Projektionsfläche der frühformalistischen Prosatheorie bereitstellte. Gleichzeitig wurde die Tendenz zur mündlichen Stilisierung in der skaz-Prosa mit dem sujetlosen Prosatypus verknüpft (Ornamentalismus plus bessjužetnost'), während traditionellere, realistische Formen der Erzählprosa den skaz vor allem zur Charakterisierung von Rednerpositionen mimetisch-realistisch einsetzten (charakternyj skaz). Im ersten Fall haben wir es mit zentrifugalen Genres zu tun, in denen die ,ornamentale“ Stilisierung von einer perspektivischen Festlegung auf einen Sprechertypus hin zu einer Montage oder einem verbalen ,Mosaik‘ (mozaika slov) dissoziiert ist. Diese Redeweise resultiert aus einem ,dissoziierten Subjekt' (rassejannyj sub"ekt), das die fiktionale, psychologische Deutung des point of view (točka

19 Schmid, Narratologie (Anm. 11), 146-162 (zur Skaz-Theorie und zur ornamentalen Prosa; HansenLöve, Formalismus (Anm. 2), 274-290; 530-537. Die klassischen Texte der formalistischen skaz-Theorie (zumal von Ejchenbaum und Vinogradov) finden sich in: Jurij Striedter (Hrsg.): Texte der russischen Formalisten, Bd. 1, München 1969. 
zrenija) in eine offene Reihe von verbalen Motiven auflöst, die eher den Prinzipien der Wortkunst als jenen der Narrativik und ihren mimetischen Ansprüchen folgt. Für Tynjanov wie Ejchenbaum regrediert dieser nicht-fiktionale, ornamentale skaz I-Typus letztlich auf die Poetik der futuristischen zaum'-Sprache mit ihrer Tendenz zur Selbstwertigkeit und zu dezentrierten Lautoberflächen. Diese Einstellung auf die mündliche Rede kann aber auch im zentripetalen Typus des skaz II fiktionserzeugend eingesetzt werden, wobei hier eine konkret nachvollziehbare, repräsentative Darstellungsperspektive markiert wird.

\section{Literatur als Explosion: Roman-Bomben und die Sprengung der Handlung}

Es ist sicherlich nicht übertrieben, Jurij Lotmans letztes Buch Literatur und Explosion als sein wissenschaftliches wie auch kulturologisches Vermächtnis anzusehen. Während in einer mit Boris Uspenskij gemeinsam verfassten Schrift die „Rolle dualistischer Modelle in der Dynamik der russischen Kultur“20 im Mittelpunkt stand, also die Homologie von binärer Kultursemiotik und dem immanenten Dualismus ihres Objekts, geht es in der Explosion-Schrift um eine Zuspitzung dieser schicksalhaften Polarisierung der russischen Kultur. Für diese gilt nicht nur eine intrinsische Spaltung, ja häretische Dualisierung, sondern auch eine spezifische Tendenz zum Bruch, zum radikalen Systemwechsel, zur Sprengung der herrschenden Verhältnisse mit dem Ziel, „Fenster in der semiotischen Schicht zu schaffen“. ${ }^{21}$ Die russische Kultur lebt ex ovo unter dem Zeichen der Explosion und der Revolution. Unübersehbar ist hier die aktuelle Situation Russlands an der Jahrtausendschwelle 2000 und nach dem Zusammenbruch des Sowjet-Imperiums. Mehr noch aber geht es Lotman um die Überwindung des kultursemiotischen Determinismus und Holismus, der herrschende Systeme scheinbar petrifiziert und durch die permanent wirksame Dynamik diskontinuierlicher Brüche abgelöst werden muss.

Lotmans im Westen erfolgreichstes Konzept - die Fundamentaldefinition des narrativen Ereignisses als ,Überschreiten einer Grenze - wird hier gewissermaßen auf die Spitze getrieben. Die Grenze der Revolution 1917 wird relativiert gegenüber jener Bombe, die im Fundament des ,Systems Russland', ja einer jeden Kultur(semiotik) unweigerlich ihrer Explosion entgegentickt und damit den Gang der Dinge taktet, eskaliert und auf anarchistische Weise bedroht.

20 Jurij Lotman, Boris Uspenskij: Die Rolle dualistischer Modelle in der Dynamik der russischen Kultur, in: Poetica Bd. 9/1 (1971), 144-166.

21 Jurij Lotman: Literatur und Explosion [Petersburg 2000], Frankfurt a. M. 2010, 233. 
Als Prätexte dieser Explosions-Apokalyptik Jurij Lotmans können zwei Texte dienen, die historisch vor und nach der Revolution positioniert sind: Andrej Belyjs Bomben-Roman Petersburg (1912), ${ }^{22}$ der sich auf die Russische Revolution des Jahres 1905 bezieht, und Viktor Šklovskijs Dokumentation Die Sentimentale Reise, die den jugendlichen Autor im Panzerwagen durch den Bürgerkrieg führt. In beiden Fällen werden die Sujets des ancien régime gesprengt, zerstückelt und in eine ,Neue Ordnung‘ gebracht: bei Belyj musikalisch-leitmotivisch, bei Šklovskij mit den Avantgarde-Prinzipien der Montage.

\section{Leitmotiv und Bomben-Prosa: Andrej Belyjs Peterburg}

In Belyjs epochalem Petersburg-Roman ${ }^{23}$ zerhackt das Ticken des Zeitzünders die politische Kriminalhandlung in ihre dramatischen Momente, die insgesamt das traditionelle Romangenre und seine nacherzählbaren Geschichten in ein vielschichtiges Geflecht von Leit- wie Leidmotiven verwandelt. ${ }^{24}$ In dieser Textur werden alle nur erdenklichen kriminologischen, politischen, ideologischen wie philosophischen und psychologischen Motivationen gegeneinander ins Treffen und letztlich ad absurdum geführt. Die von den Wagner-Opern inspirierte Leitmotivtechnik des verklingenden Symbolismus trifft hart auf die brutale Montage-Technik der im zweiten Jahrzehnt nach 1900 fast gleichzeitig erwachenden Avantgarden in Literatur und Kunst.

Nikolaj Apollonovič Ableuchov, Sohn und Vatermörder des Bomben-Romans, trägt schon im Vornamen eine dionysische Sprengkraft, die sich gegen die Schande, ja den geradezu physischen Ekel richtet, welcher aus seiner paranoiden Sicht an der leiblichen Herkunft von einem verhassten Vater-Körper haftet. Nikolaj erfährt an sich selbst die Zerfleischung des Dionysos, er fühlt in sich „ein aufgedunsenes Monstrum“, das seinen Körper grotesk „umstülpt“ und zum Platzen bringt: „ich zersprang wie eine Bomb...“": Ebenso wie das Wort „Bomb/e“ selbst zerstückelt wird, ist der ,Bombensatz“ fragmentiert und „In Stücke!..“ zerlegt. ${ }^{25}$ Der ganze Romantext ist „gestottert“, seine Syntax mit all ihren Aposiopesen und elliptischen Satz-Stummeln flackert durch den Kopf des Lesers wie das Gezappel der Stummfilme jener Jahre, die auch in Petersburg zur Stadtfolklore gehörten und der Prosa ein ungeahntes Tempo aufdrängten.

22 Andrej Belyj: Petersburg, übers. von Gabriele Leupold, Frankfurt a. M. 2005.

23 Eine ausführliche Fassung dieses Kapitels in: Hansen-Löve, Schwangere Musen (Anm. 6), 482-498. 24 Vgl. auch V. Šklovskij: Ornamental'naja proza. Andrej Belyj [Ornamentale Prosa], in: ders.: O teorii prozy, Moskau 1929, 205-225.

25 Belyj (Anm. 22), $388 \mathrm{f}$. 
An einer scheinbar nebensächlichen Stelle stößt der Leser - ebenso wie Nikolaj Apollonovič - auf jenes hochexplosive Bündel, dessen Inhalt er nur zu gut erahnen kann:

Diesmal wunderte sich Nikolaj Apollonowitsch...

- „Das ist wahrscheinlich Literatur?...“

- „Nun, nein...“

.$^{26}$

Ja, es ist eine Bombe im direkten und übertragenen Wortsinn: Die Bombe ist nichts anderes als ein ,Stück Literatur' und umgekehrt: Der Roman selbst ist die Bombe, mit der sich die Romanfiguren gegenseitig verfolgen und dann - nicht einmal das gelingt in die Luft sprengen wollen. So wird aus einem Bomben-Roman eine Roman-Bombe und diese ist so schlecht verpackt, dass die Leser ebenso wie die Mitverschwörer permanent befürchten müssen, der fatale Inhalt des „Bündels“ könnte unabsichtlich und vorzeitig ,auffliegen“. Zugleich ist eben dieser „Inhalt“ das narrative Objekt der Begierde des Romans, dessen Kriminalspannung durchaus unprofessionell, absichtlich-absichtsvoll dilettantisch in Szene gesetzt wird. Es ist ja ,nur' Literatur, die sich in jenem Bündel verbirgt, aus dem es letztlich besteht. Offen bleibt, ob das ganze Geschehen überhaupt einen Inhalt hat, ein solcher ist und daher in der Lage wäre, die auseinanderdriftenden Inseln und Ufer der Romangeographie zusammenzuhalten und dabei die Motivblöcke mit ihren Zugbrücken aneinander zu ketten.

\section{Šklovskijs Sentimentale Reise in den Bürgerkrieg}

Das hoch Ironische von Šklovskijs Sentimentaler Reise ist die krasse Diskrepanz zwischen den blutigen Bürgerkriegserlebnissen des durch die Kampfzonen ratternden auktorialen Chauffeurs und die Reflexionen dieses Erzählens vom ,Schreibtisch` aus. Das Erzählen wird durch permanente Unterbrechungen zerstückelt und zerfetzt durch die den gesamten Text leitmotivisch durchlaufenden Hinweise auf jene Bombe, die letztendlich in den Händen des Autors explodieren sollte. Wir erinnern uns hier an die tickende Bombe in Belyjs Petersburg-Roman, wo der Sprengkörper zuletzt doch nur in einer Toilette ,losgeht‘: ${ }^{27}$ naturgemäß ,nach hinten'. Bei Šklovskij liegt das Ganze von Anfang an und wortwörtlich auf der Hand.

\footnotetext{
26 Ebd., 113.

27 Die Toilette taucht auch an anderer Stelle auf (ebd., 177 f.), sie vermittelt dort mythopoetisch als „Wasser-Klosett“ zwischen der Kanal- und Unterwelt Petersburgs und der Steinwelt der Behausungen, indem eine vertikale Verbindung zwischen beiden Sphären, ebenso wie zwischen Ober- und Unterleib, apollinischer und dionysischer Sphäre hergestellt wird. Dass solchermaßen die analen Produkte entsorgt werden, erweist sich als eine ebenso praktische wie kaum gewürdigte Konsequenz.
} 
Für die jugendlichen Vertreter des russischen Formalismus gab es nicht nur keine Differenz zwischen Literaturtheorie und -praxis, zwischen Objekt- und Metaebene im Schreiben und zwischen Wissenschaft und Existenz im literarischen Dasein. Das eigene Schreiben und das Reflektieren darüber sollte zu einem Anti-Roman verschränkt werden, zu einem Genre ohne durchgängige ,Handlung‘ bzw. ,Sujet‘ und ohne die Manipulationen einer narrativen Fiktionserzeugung und damit eines - wie man meinte - falschen literarischen Bewusstseins. Auf diese Weise wird das theoretische Konzept, konkret die Prosatheorie des Formalismus, in ein und demselben Genre thematisiert und zugleich konstruktiv realisiert, auf der narrativen Ebene inszeniert und auf der metapoetischen Ebene reflektiert. Dass dabei der von Šklovskij für die russische Prosatheorie ${ }^{28}$ neu entdeckte „typischste Roman der Weltliteratur“ - Laurence Sternes Tristram Shandy ebenso wie seine Sentimentale Reise - Pate stehen sollte, gehört gleichfalls zur Ironie der Literaturgeschichte und ihrer leichtfertigen Anspielungskunst, die sich um den Ernst der (historischen) Lage ebenso wenig kümmert wie ein pikaresker Held des Sentimentalismus oder der Epoche der Schelmenromane.

Nicht ganz grundlos sah Nabokov in den Romanen Belyjs die bedeutendsten Hervorbringungen der russischen Romankunst des Jahrhunderts; manche verglichen Belyjs Prosa gar mit jener von James Joyce. ${ }^{29}$ Für unsere Zwecke mag es genügen, sie in eben jene antigenerische Linie zu stellen, die - um Šklovskijs Schreibfigur aufzugreifen - nicht vom Vater auf den Sohn, sondern vom Onkel auf den Neffen überging. Diese Evolution verlief in eben jenen ,Sprüngen', wie sie auch textintern von den Sujets vollzogen wurden: mutwillige Wendungen, verrückte Rück- und Übergriffe, Verschiebungen der Wortmassen und Motivkomplexe, rhythmisch-prosodische Verknüpfung von Leitmotiven, deren narrative (Kon-)Sequenz ersetzt wird durch lautliche, ja kalauernde Assonanzen und Wortspiele.

Eines der Hauptverfahren bei dieser Tendenz zur Sujetlosigkeit war die von Sterne adoptierte Abschweifungstechnik und die damit einhergehende Sprengung der narrativen und deskriptiven Motivblöcke in minimale Absatzfragmente, die kontrastiv und leitmotivisch zugleich miteinander verzahnt wurden. Diese Schreibtechnik hat es zuvor in der dithyrambischen Prosa Nietzsches gegeben, dessen stilistischer Einfluss auf die russische Erzähltechnik unübersehbar war - ebenso aber in der fragmentarischen Schreibweise Vasilij Rozanovs, die Viktor Šklovskij in ihrer revolutionären Bedeutung für die zeitgenössische Prosa(theorie) entdeckt hatte. ${ }^{30}$

28 Hansen-Löve, Formalismus (Anm. 2), 530 ff.; Schmid, Ornamentales Erzählen (Anm. 3); Šklovskij, Theorie der Prosa (Anm. 17), 131-162; zur Poetik von Šklovskijs Sentimentaler Reise vgl. Anna Dwyer: Russland wieder beleben. Literatur, Theorie und Imperium in Viktor Šklovskijs Schriften aus dem Bürgerkrieg, in: Kakanien revisited 12/11 (2009), 1-15.

29 Vgl. Alexander Woronzoff: Andrej Belyj's Petersburg, James Joyce's Ulysses and the Symbolist Movement, Bern u. a. 1982.

30 Viktor Šklovskij: Rozanov, Petrograd [1921], in: Teorija prozy, Moskau/Leningrad 1925, 162-178. 
Diese Abschweifungstechnik verlagerte Šklovskij aus der immer wieder anklingenden Prosa Sternes auf die Baustellen von Montagen, die eher destruktiv als konstruktiv auf einen hoch empfindlichen sophisticated reader einwirken sollten. Nicht zufällig waren es ja gerade die Formalisten, die diese konkrete De-Konstruktion der Motivsequenzen in der Wortkunst auf vergleichbare Techniken in eine von ihnen geschaffenen Filmpoetik (Poètika kino, 1926) übertragen konnten. ${ }^{31}$ Auch hier war die Verfremdungsästhetik aus der Malerei und Literatur in die im Entstehen begriffene Stummfilmkunst projiziert worden, wobei sich die Verfahren der Montage als eine ungemein kreative Technologie der antigenerischen Textproduktion erwiesen. Gleichzeitig werden die rezeptionsästhetischen Grundsätze des frühen Formalismus als Theorie wie als Existenzform vorgeführt. Dabei erwächst aus dem antigenerischen Status des auktorialen Helden und seiner Outsider-Perspektive eine soziale, politische, ideologische wie psychologische Extremposition, die aus der ästhetischen und poetischen Dimension der Verfremdung eine existentielle der Entfremdung macht.

Diese anekdotisch aufblitzenden Signale einer egalitären Haltung der Protagonisten angesichts der auf Polarisierung und Zuspitzung fixierten Bürgerkriegsszene verfestigt sich zum Habitus einer auf Äquivalenz und Dissoziation bedachten Beobachterposition, die eine monolithische Autorschaft ebenso zerrüttet wie die Identitäten agierender und agitierender Helden. Eben das Leitmotiv der Bombe, das wenige Jahre zuvor Belyjs Petersburg-Roman mit seinem fatalen Ticken in der Sardinenbüchse schrecklichen Inhalts getaktet hatte, dieses Ticken perforiert auch das Absatzgestolper der Sentimentalen Reise, die der Bahn des „fallenden Steins“ folgend auf eine multiple Explosion zusteuert. Das zentrale konstruktive wie existentielle Motiv lautet: „Wenn man fällt wie ein Stein, darf man nicht denken; wenn man denkt, darf man nicht fallen. “32

In genau dieser chiastischen Verschränkung manifestiert sich der Knoten, das double bind des homo theoreticus, der die traditionellen Handlungsträger und ihre väterlichen Autoren paralysiert, sodass die manipulierten Verknüpfungen zwischen den Handlungszellen in einer empirischen wie einer überbewussten Doppelbeleuchtung aufscheinen: Der Autor fragt sich, wie er jenen Teil seines Wesens, der denkt, der Kunsttheorien entwickelt, der schreibt, reflektiert, sich erinnert, mit jenem anderen Teil vereinen kann, der handelt - oder genauer, der von Kräften beherrscht wird, die nicht in der Macht des Denkens liegen: „Ich bin Professor am Institut für Kunstgeschichte, Begründer der formalen (oder morphologischen) Methode

31 Vgl. die deutsche Ausgabe: Poetika Kino - Theorie und Praxis des Films im russischen Formalismus, hrsg. Wolfgang Beilenhoff, Frankfurt a. M. 2005 (mit Texten von Šklovskij, Ejchenbaum und Tynjanov).

32 Šklovskij, Sentimentale Reise (Anm. 2), 191. Zitate aus dieser Ausgabe werden im Folgenden direkt im Lauftext nachgewiesen. 
in der russischen Literaturwissenschaft, ich bin ein fallender Stein. Ich bin wie eine Nadel ohne Faden, die durch den Stoff gleitet, ohne eine Spur zurückzulassen. “ (312); „[D]ie Schwerkraft der irdischen Gewohnheiten zog den Stein des Lebens, den die Revolution waagrecht geworfen hatte, auf die Erde herunter. Der Flug wurde zum Fall.“(332)

Während Mandelstam noch die Spitze der Nadel und ihr Spitzenprodukt zu feiern wusste - immerhin nannte sich seine Gruppe Akmeisten, also ,Spitzenkünstler ‘ -, gibt es bei Šklovskij keine rissfesten Texturen mehr. Ja, diese sind gar nicht vorhanden, die Nadel hat den (roten) Faden verloren und zieht keine ,Handlungslinien“ nach sich: „Aber ich bin ein Kunsttheoretiker, ich bin ein Stein, der beim Fallen in die Tiefe schaut, ich weiß, was eine Motivierung ist.“ (248) Genau das aber sollte der Fallende vermeiden: den Blick in die Tiefe; um an der Oberfläche zu bleiben, muss er die Augen offen halten für das, was sich zeigt und was sich gleichsam selbst begründet: „Ich kann mich treiben lassen, ich kann mich beliebig verwandeln, ich kann sogar zu Eis werden oder zu Dampf, ich passe in jeden Stiefel.“ (234)

Aus den wissenschaftlichen Persönlichkeiten der Formalisten werden solchermaßen literarische bzw. fiktionale Figuren; die formalistische Kritik am generischen Biografismus erfährt dabei eine ironische, ja selbstparodistische Note: „Ejchenbaum sagt, das revolutionäre Leben unterscheide sich hauptsächlich dadurch vom normalen, daß wir jetzt alles viel stärker empfinden. Das Leben ist Kunst geworden.“ (373) Diese Behauptung bezieht sich auf eine der Grundthesen der frühformalistischen Verfremdungs-Theorie, deren innere Spannung und Dynamik aus einer Intensivierung der Sensitivität, der Empfindungsfähigkeit resultiert, wodurch die konventionellen Wahrnehmungs- und damit auch Wertungsnormen entautomatisiert, verschoben und neu gestaltbar werden.

Wie in Dostoevskijs Jüngling-Epilog im Zusammenhang mit dem Familienroman à la Tolstoj wird gerade bei Šklovskij die Anormalität der revolutionären Epoche rückwirkend mit jener der Kunstrevolution assoziiert: „Das Leben treibt in abgerissenen Bruchstücken dahin, die verschiedenen Systemen angehören. Einzig unsere Kleider, nicht unser Körper, verbinden die voneinander isolierten Augenblicke des Lebens.“ (253-254) Das Sujet - oder genauer: die fabula - des Lebens, der biographischen Verläufe, der kollektiven Narrative, hat sich in zahllose Bruchstücke zerlegt, die keiner generischen Kohärenz mehr folgen und so aus der konventionellen ,Erzählung` der Kultur herausgefallen sind. Wenn es für Dostoevskij im Epilog zum Jüngling keine traditionellen Romane mehr geben kann, weil ihnen das erforderliche Material - eine Familiengeschichte - fehle, dann gibt es für Šklovskij das Romangenre in seiner generischen Form nicht mehr, weil die dazugehörigen Lebenstexte völlig inkohärent geworden seien.

Gleiches gilt für den antigenerischen Helden, der - nolens volens unter den herrschenden Bedingungen - nur noch als ,Schnittpunkt' externer Kraftlinien figuriert, die eindeutig außerhalb seiner Autonomie und Eigengesetzlichkeit (wohl auch der Kunst und der Romanwelt) liegen. Diese ,Existenzialisierung' und letztlich auch 
Sozialisierung der völlig gegenläufigen Konzepte der Verfremdungs-Theorie ${ }^{33}$ reduzieren die Position des Autors wie der Figuren scheinbar wieder auf die alte Formel, dass die externen Kräfte, das Realitätsprinzip und die gesellschaftlichen wie politischen Strukturen (bzw. ihre Auflösung) verantwortlich sind für die Zersplitterung der narrativen Sequenzen und ihrer innerkünstlerischen Motivierungen.

Die Verfechter traditioneller Erzählgenres und Kulturnormen hatten in den 20er Jahren alle Hände voll zu tun, diese Dynamik der Montagekultur insgesamt zu brechen oder jedenfalls in die gewohnten Prokrustesbetten einer linearen Narrativik - späterhin des Sozialistischen Realismus - einzusargen. Dies ist ihnen freilich nur unter Zuhilfenahme staatlicher Instrumente der Disziplinierung und Liquidierung ganzer Kunstzonen und ihrer Protagonisten gelungen, so sehr freilich, dass schließlich eine zutiefst generisch orientierte Kulturreaktion der Stalinära die alten Schablonen des literarischen Personenkults wie der hoch riskanten auktorialen Textverantwortung triumphieren ließen. Das traditionelle Paradigma einer autoritären, ja autokratischen Autor-Held-Relation konnte so Jahrzehnte lang künstlich bzw. gewaltsam am Leben gehalten werden, sodass ganze Generationen von literarischen Helden - zusammen mit ihren Lesern - an die alten narrativen Machtverhältnisse gefesselt blieben.

Šklovskijs epochemachender Artikel Die Kunst als Verfahren (Iskusstvo kak priem, 1919) verweist auf einen Hintersinn des sich im Chaos des Bürgerkriegs ver-fahrenden Automobils: Im Unterschied zur Kutschenwelt des Realismus (Gogol), der Eisenbahnprosa (von Tolstoj zu Platonov und Pasternak) sowie der Poetik des Fliegens (im Futurismus), bewegt sich Šklovskij in einer Auto-Mobil-Welt, die mit der Vorstellung einer multiplen, von allen narrativen Schienen und Pisten befreiten Schreib- und Denkweise der formalistischen Prosa-Avantgarde voll übereinstimmt. Der Autor bewegt sich wie der Chauffeur planvoll und frei zugleich in der Kartographie der Handlungslinien und Sprachregeln, er kann jederzeit von der Magistrale der narrativen „Hauptlinie“ abzweigen und eben jener Lust an der Abschweifung frönen, die den antigenerischen Roman insgesamt auszeichnet.

Das Automobil gehört allen (,Omnibus`), es ist gewissermaßen demokratischer als die Bahn oder gar die Kutsche bzw. der Schlitten. Der Automobilist ist eben ein ,SelbstBeweger', der den Treibstoff des Lebens, das Faktenmaterial der vorliterarischen Welt in seinem Motor verbrennt und zu jener Energie umwandelt, mit der sein Fahrzeug in Bewegung gehalten wird. Der Autor sitzt im Auto am Lenkrad, während die Leser im Fond sich ordentlich durchschütteln lassen müssen. Hie und da werden aber sie selbst

33 ,Entfremdung، (otčuždenie) markiert immer eine sozial-psychologische Bewusstseinslage des Subjekts, das seinem eigen(tlich)en Wesen aufgrund von ,Automatisierung' von Arbeits- und Lebensprozessen nicht gerecht wird; die (ästhetischen, poetischen, noetischen) Verfahren der ,Verfremdung ‘ (ostranenie) bewirken genau das Gegenteil: Steigerung der Sensitivität und der Reflexivität der Wahrnehmungs- und Erkenntnisprozesse. Vgl. Hansen-Löve, Formalismus (Anm. 2), 19 ff.; ders., Die Theorie der Verfremdung im russischen Formalismus, in: Hermann Helmers (Hrsg.), Verfremdung in der Literatur, Darmstadt 1984, 393-427. 
ans Steuer gelassen, um dem Eindruck zu erliegen, selbst den Wagen zu lenken und auto-mobil zu sein. Šklovskijs Gefährt war freilich in einem Bürgerkrieg unterwegs und daher gepanzert, wie der Panzerzug Vsevolod Ivanovs in dessen gleichnamigen Bürgerkriegsroman, ${ }^{34}$ der freilich eher in der ornamentalen Sphäre der Serapionsbrüder aktiv war. Šklovskij war zweifellos ein Mann der Tat, der Wort-Tat - sicherlich kein Schreibtischtäter, der sich auf die Fiktionslust eines Publikums verlassen wollte. In seiner Prosa(theorie) ist die antigenerische Botschaft des englischen Sentimentalisten zum Medium einer neuen, erfrischenden Prosa aufgestiegen und aus dem literarischen Ausnahmezustand zu einer - wenn auch immer wieder bekämpften - Norm erwachsen.

Die zentrifugalen Kräfte der aus den Fugen geratenen Zeiträume sind mit der Dissoziierung homogener fabula-Sequenzen zu scharf konturierten Textblöcken montiert, die narrativen Konsequenzen explodieren zu den disiecta membra rhythmisierter Absätze, die im Takt der modern times und ihres kinematografischen Zeitraffers aufblitzen. Die ,gestufte Konstruktion“ finden wir in Belyjs später Lyrik ebenso wie in seiner Romanprosa. Der ,Absatz‘, die montierten Zeilen und ihre Fragmente ersparen eine erklärende, motivierende Überleitung zwischen den Segmenten; der Leser bleibt an den Rissen und Sprüngen der Montageelemente hängen und folgt gebannt dem Krachen und Knacken ihrer Zusammenbrüche. Da stoßen minimale, zu Idiomen komprimierte Anekdoten auf die Scheinwerfergarben hochtheoretischer Reflexionen, die sich in das schütter gewordene narrative Geflecht hineinfressen:

Auf einer meiner Fahrten traf ich im Zug einen Artilleristen.

Wir kamen ins Gespräch.

Er war mit seinem 7,5-cm-Geschütz schon viele Male in Gefangenschaft geraten, bald bei den Weißen, bald bei den Roten.

Er sagte: „Ich weiß nur eins: Meine Aufgabe ist es, mein Ziel zu treffen.“ (221)

Das Bomben-Motiv taktet also den Text, bricht immer wieder scheinbar völlig unmotiviert und bedrohlich hervor, so etwa als die „in der Hand explodierende Bombe“, die eigentlich entschärft werden sollte: „Nachts schrie ich im Schlaf, ich träumte, eine Bombe explodiere in meiner Hand.“(374) Der „falsch zusammengesetzte Leichnam“ (182) erinnert an den zusammengeflickten Montagetext, der schließlich unter der Hand des Autors in die Luft fliegt und sich in seine Bestandteile zerlegt (182, 192): „Ich träumte oft in der Nacht, daß mir in der Hand eine Bombe explodiert. - So etwas habe ich einmal erlebt.“ (248)

Tatsächlich beschrieben wird der immer wieder antizipierte Vorfall erst gegen Ende der Sentimentalen Reise; so wird aus einer postponierten Exposition eine immer wieder hinausgezögerte Explosion, die sich mit dem Motiv eines reflektierten Fallens überschneidet: „Denken macht zerstreut. Ich habe mich aus Zerstreutheit in

34 Vsevolod Ivanov: Bronepoezd, Moskau 1922, 14-69, deutsch: Panzerzug, Frankfurt a. M. 1970, 14-69. 
die Luft gesprengt.“ (298) Das im Formalismus zelebrierte ,zerstreute Subjekt“ (rassejannyj sub"ekt) markiert hier auf ironische Weise sowohl Ursache als auch Wirkung einer gebremsten, konstruktiv verzögerten Destruktion, während als der eigentliche Grund des Konzentrationsmangels das Schreiben selbst figuriert, das seinen Autor im entscheidenden Moment abgelenkt hatte, da er an sein Buch „Das Sujet als Stilphänomen“ denken musste (ebd.). In dieser Schrift aus dem Jahr 1917 sind eben jene Verfahren theoretisch auf denjenigen Punkt gebracht, der als locus saliens des Lebensund Schreibtexts funktioniert: „Daß ich eines Tages in die Luft fliegen würde, stand ja von vornherein fest.“(302)

Wie Evgenij Zamjatin in seinem utopiekritischen Wir-Roman hat auch Šklovskij mit seiner Wie-Prosa die Tendenzen eines totalitären Denkens in Ost und West vorwegnehmend kritisiert, ja in ihrer diktatorischen Bedrohung früh erkannt und mit den Mitteln der analytischen ,Entblößung der Verfahren“ verfremdet und entlarvt. Indem das ,Wie‘ des Schreibens und Schaffens zum eigentlichen ,Helden` der Literaturwissenschaft erhoben wurde, ${ }^{35}$ sollten auch die traditionellen Erzählfiguren samt ihren Autoren aus ihrer dominanten Stellung vertrieben, de-generiert und defiktionalisiert werden. Die formalistische Verfremdungs-Ästhetik war damit viel weniger eine ,formale‘, auf die äußeren Dekorationen einer Komposition fixierte Lehre, sie war vielmehr die ,Methode، - analog zu jener der Psychoanalyse Freuds -, die keine Wissenschaft sein wollte und keine Schule, sondern eine Sicht- und Schreibweise. Sie sollte den ödipalen Machthaber und seine fatale Natalität durch einen emanzipierten Poeten ersetzen, dessen Artefakte ex negativo auf die deformierte Vorwelt gerichtet sind. Es ging um das „Machen des Dinges“, ${ }^{36}$ genauer: das Sichtbar- und Hörbarmachen, das Neuentdecken der ursprünglichen Dinglichkeit unter der abgenützten Oberfläche der Gegenstandswelt und ihrer Familienromane, die so taten, als wären nur sie rechtmäßige Eingeborene und Erben der Literaturwelt.

Im Bürgerkrieg der Literatur hatte ein staatlich verordneter Realismus gesiegt, den es in dieser platten Form auch nicht im verhassten 19. Jahrhundert gegeben hatte. Wie in den meisten Fällen von Reaktion und Restauration, die im Kern von Revolutionen lauern, wurden auch hier ganze Generationen in eine Welt vor der Moderne zurückgebombt. Während die Kulturrevolutionäre über das Vorgestern ins Übermorgen springen wollten, implodierte die postrevolutionäre Welt in einem Gestern, dem kein guter Morgen gegönnt war, eher ein gähnendes Morgen-Grauen, vor dessen

35 So Jakobson: Die neueste russische Poesie, in: ders.: Poesie der Grammatik und Grammatik der Poesie. Sämtliche Gedichtanalysen. Kommentierte deutsche Ausgabe, Bd. I, Poetologische Schriften und Analysen zur Lyrik vom Mittelalter bis zur Aufklärung, gem. mit Sebastian Donat hrsg. von Hendrik Birus, Berlin/New York 2007, 1-123, hier: 16.

36 Vgl. den Tagungsband: Anke Hennig/Georg Witte (Hrsg.): Der dementierte Gegenstand. Artefaktskepsis der russischen Avantgarde zwischen Abstraktion und Dinglichkeit, Wiener Slawistischer Almanach, Sonderband 71, Wien/München 2008. 
Undurchdringlichkeit sich die fröhlichen Gestalten der Serapionsbrüder und der frechen Formalisten so erfrischend und bis heute inspirierend abheben.

Es macht Mut, die Literaturgeschichte ein Jahrhundert zurückzublättern, um einen Blick in jene Präsenz zu erhaschen, die eine genialische Generation von Prosaikern einige wenige Jahre zwischen den beiden Abgründen erkämpft hatte: zwischen der fatalen Handlungsschwäche des 19. Jahrhunderts - und der nur zu effektiven sowjetischen Zement-Trommel, die dann auf Jahrzehnte einen ganzen Literatur-Kontinent zubetonieren sollte. Auf sie zielte die Sprengkraft der narrativen Sujets jener jungen Prosa um 1920, deren Explosionen wir noch aus der Ferne hören: Sprengungen versteinerter Narrative und das Knattern fröhlicher Feuerwerke der befreiten Sprache. 